\title{
Social Business - A New Dimension for Education and Training in Organizations The EToW Model
}

\author{
Maria João Ferreira ${ }^{1,2}$, Fernando Moreira ${ }^{1,3}$ and Isabel Seruca ${ }^{1,2}$ \\ ${ }^{1}$ DICT, Portucalense University, Porto, Portugal \\ ${ }^{2}$ ISTTOS, Centro Algoritmi, Minho University, Guimarães, Portugal \\ ${ }^{3}$ CLEGI, University Lusiada de Vila Nova de Famalicão, Vila Nova de Famalicão, Portugal
}

Keywords: $\quad$ Social Business, Social Tools, Training.

\begin{abstract}
Organizations have suffered a large (r)evolution at the social, economic and technological levels. A change of paradigm in what comes to information systems and technologies (IST) used in the day-to-day life of every citizen, by itself, does not sustain such a transformation; it is therefore necessary a change of culture and behaviour. The use of IST in an appropriate and integrated way with the organization's processes will depend on an individual and collective effort. The younger generation, familiarized to sharing, often through mobile devices, personal information on social networks, enters the job market looking for similar tools. These social tools allow the production, sharing and management of information and knowledge within the organization between peers and other stakeholders, eliminating the barriers of communication and sharing. Taking advantage of these technologies for the education and training of organization' employees within the context of Social Business, in particular concerning nomadic workers, requires a comprehension exercise in how to demonstrate their usefulness with regard to the creation, access and sharing of contents in a safe way. To this end, this paper proposes a model for the $2^{\text {nd }}$ layer - Education and Training of Organizational Workers - of the mobile Create, Share, Document and Training (m_CSDT) framework.
\end{abstract}

\section{INTRODUCTION}

It is widely acknowledged that organizations have suffered a large (r)evolution at the social, economic and technological levels, with particular predominance in the last 10 years, where the traditional barriers of transferring information and knowledge silos have been progressively eliminated. Finding experts and knowledge within an organization is now easier through Social Business. Social Business (IBM, 2013) can be defined as the ability of an organization to share information, produce knowledge collaboratively, manage knowledge, eliminate communication and sharing barriers, accelerate business processes, approaching the business partners, namely suppliers and customers, and create innovative products, services and business models. It is thus essential that such products, services and models are created and properly documented, managed and shared.

A change of paradigm in what comes to the use of information systems and technologies (IST) in the day-to-day life of every citizen, by itself, does not sustain such a transformation; it is also necessary a change of culture and behaviour. On the one hand, the use of IST in an appropriate and integrated way with the organization's processes will depend on an individual and collective effort, which may be called "collective leadership" (Friedrich et al., 2009). On the other hand, the younger generation, accustomed to sharing, often through mobile devices, personal information on social networks, enters the job market looking for similar tools. These new "social tools" allow the production, sharing and management of information and knowledge within the organization between peers and other stakeholders.

Therefore, we may infer that Social Business is much more than just collaboration and sharing, since the IST that are currently available allow the organizations' processes to be more dynamic, more "social". 
Such evolution is also observed in the context of mobile devices. These are becoming ubiquitous and mobile learning/training is increasingly becoming an option. Furthermore, the use of mobile devices for personal and professional use will grow continuously over the next years.

Taking advantage of these technologies for the education and training of employees in organizations within the context of Social Business, in particular for nomadic workers, requires a comprehension exercise in how to demonstrate their usefulness with regard to the creation, access and sharing of targeted contents in a safe way. In this paper we propose a model for the $2^{\text {nd }}$ layer - Education and Training of Organizational Workers (EToW) - of the mobile Create, Share, Document and Training (m_CSDT) framework described in (Ferreira, Moreira \& Seruca, 2014).

The rest of the paper is structured as follows: Section 2 describes the contribution of social business as a new trend for organizations. Section 3 provides a summary of the use of social media tools in several contexts. Section 4 discusses the evolution of social media training in organizations, identifying the main trends. Section 5 presents the contribution of our model to support the education and training of organizational workers. Finally, Section 6 concludes with a summary of our achievements and directions for future work.

\section{A NEW TREND FOR ORGANIZATIONS - SOCIAL BUSINESS}

\subsection{Social Business}

The concept of social business was firstly associated with Yunus work (Yunus, 2007), where the concept involved both the economic and social perspectives; that is, social business was supposed to solve a social problem and to achieve financial sustainability rather than to generate profits. A new perspective was introduced by Kim (2012) in a blog post where the concept was applied to evolutionary marketing and technology strategies. Social Business is a recent but popular trend that is revolutionizing organizational work and generating value for all of its elements, i.e. employees, customers, partners and suppliers. It means that all departments in an organization integrate their social capabilities into traditional business processes to change the way of working in order to create value.
A Social Business organization uses social software technology to communicate with its rich ecosystem of customers, business partners and employees. In order to achieve that with success, long term goals are needed to be set, i.e. profitability, productivity, competitive position, employee's development, employee relationships, public responsibility, and technological leadership.

Hagel et al. (2010) claim that "each of us, individually and together, are now, for the first time in history, in a position to collaborate in a complete reimagination of our biggest private and publicsector institutions that will eventually remake society as a whole". Following this imperative, organizations must be open to be supported by social business, which constitutes a shift in how people work, moving from hierarchies to networks. Nowadays, complex work is the most valuable, as well as the type of work that cannot be automated or outsourced. It is work that requires creativity and passion. Doing complex work in networks means that information, knowledge and power no longer flow up and down.

The work underlying social business can only be achieved through social learning; Jarche (2011) synthesized this idea into ten statements and argues that training courses are artefacts of a time when information was scarce and connections were few, a time that has passed.

\subsection{Integrating Social Business into an Organization}

The future of organizational work is social, collaborative and mobile. The introduction of social business into an organization requires important changes in the way its collaborators work within the whole organization structure (Cortada et al., 2012). According to Cortada et al. (2012), in order to integrate social business in the core of an organization three key issues must be addressed: (i) Organizations need to consider how to incorporate social metrics in themselves and in their processes; (ii) Organizations need to understand and manage the risks associated with the integration of social business; and (iii) Organizations need to manage change, which is a fundamental requirement to undertake with success social business practices.

Moreover, in SideraWorks (2013) seven dimensions are proposed that must be questioned and defined: (1) vision and goals; (2) cultural readiness; (3) organisational structure; (4) social strategy; (5) communication; (6) social technologies; and (7) training and education. 
In this context, training and education (the seventh dimension) should allow knowledge to flow in the organization. The primary function of training and educating professionals in the networked organization is to connect and communicate based on three core processes: (i) Facilitating collaborative work and learning amongst workers, especially as peers; (ii) Sensing patterns and helping to develop emergent work and learning practices; (iii) Working with management to fund and develop appropriate tools and processes for workers.

Learning through connection and collaboration through the use of various social media tools happens, and is growing, in the workplace, with or without being formally recognized. The integration of learning mediated by these tools is of considerable interest to the organizations on the effects that these have on learning and organizational development.

\subsection{Social Business Contributes for a More Competitive Organization}

During the last few years, social technologies allowed social interactions with the speed and scale of the Internet. Whether discussing consumer products or organizing political movements, people around the world constantly use social media tools to seek and share information. And, in this context, business is changing their behavior and social media becomes an important business tool (McKinsey and Company, 2012).

The value of organizations that use social media tools is determined by how they are harnessed to create value for them (Majchrzack et al., 2009). Martin and van Bavel (2013) present a set of potential benefits, i.e., tangible and intangible gains, when the organizations use social media tools, which they classified in terms of a) employees' use of the technologies, b) customer engagement activities, and c) external partner activities.

According to these authors, the benefits in these three dimensions were summarized as follows. For employees: (i) Fast access to organizational knowledge; (ii) Fast access to internal expertise; (iii) Greater collaboration opportunities; (iv) Reduced internal communication costs; (v) Improved training processes and (vi) Improved recruitment of new employees. Regarding customer engagement: (i) More effective branding, stronger brand loyalty, and improved marketing, including stronger customer dialog; (ii) Ability to mine and analyze customer data more effectively; (iii) Ability to personalize marketing activities based on customer interests; (iv)
Improved customer service and support; (v) Better product development; (v) Increased customer satisfaction, and (vi) Reduced marketing costs. Finally, for external partners: (i) Fast access to external knowledge and improved information sharing; (ii) Reduced external communication costs; (iii) Improved collaboration and innovation opportunities, and (iv) Exploiting new marketing opportunities.

\section{THE USE OF SOCIAL MEDIA TOOLS}

\subsection{The Use of Social Media Tools in Organizations}

Social media tools are supposed to inflict a sharp transformation in the employees' private and professional lives. Through careful observation of the use of such tools, it is widely acknowledged that they are vital in the process of communication in society today. Nowadays, we live in a world where people have opportunities to connect, communicate and collaborate in ways that were previously inconceivable. Only in the last decade, there has been an overwhelming change in the way people use the Internet (Everson et al., 2013).

With the large number of young people entering the job market with skills in the use of social media tools, organizations cannot ignore this influence on the habits and skills of their employees.

The work discussed by Myers (Myers, 2013) points out a study based on 3500 interviews made to businessmen, from which was concluded that $83 \%$ of the organizations involved in the survey already use social media tools in several activities. Another interesting finding relates to mobility, where $48 \%$ of the organizations that participated in the survey indicate that employees have access to mobile devices and $65 \%$ of these use at least one mobile social tool. The same study showed that organizations, on the one hand, are recommending increasingly mobile access to their employees and, on the other hand, begin to use social media tools as early as possible in order to benefit from the combination of the both.

According to Cara (2010), more than $70 \%$ of what is learned throughout life and work is learned informally and socially. In this work some interesting results are presented. The fact that social media tools constitute a vital tool for education and training of employees is highlighted with $90 \%$ of 
respondents supporting this statement, $81 \%$ of respondents feeling that social media tools offer very interesting opportunities for employees' learning and $98 \%$ of respondents agreeing that social media tools are changing the way employees are learning and accessing information.

Jarrahi \& Sawyer (2013) mention that what is known so far on the use of social media tools at work is based on studies focused on the use of a social media technology by itself. These studies provide useful information about some organizational implications of its use, but do not consider how technologies can be used together.

\subsection{The Use of Social Media in Education}

Today's students and future professionals are digital natives. They grew up with access to computers and the Internet, and belong to a generation whose vocabulary includes words like blog, Facebook, among others. In addition, they have a decade or more of experience in using social media tools to share information and make connections. Thus, students who grew up with this type of social media tools are the ones providing answers to new industries when they leave the university.

Some studies have been conducted to verify the importance that social media tools have in teaching. For example, Young (2010) refers that about 750 teachers participated in a conference on emerging technologies for computer-based learning. At that conference teachers defended the use of Facebook in education. They concluded that this medium has advantages over typical LMS (learning management systems), since many students already know how to use Facebook, which saves time in the classroom. Yet another study (Wankel and Wankel, 2011), shows that university leaders began to use tools such as Facebook, Twitter and YouTube to manage issues related to the day-to-day of institutions and public relations, while some university members use social media tools for teaching and research.

Some authors, such as Dunlap and Lowenthal (2011), believe that the use of social media technologies, on which students are already familiar can help and support lifelong learning, because they: (i) develop the student's autonomy, responsibility and intentionality, (ii) encourage reflection, (iii) allows the participation in communities of practice, (iv) encourage collaboration and sharing, and (v) provide motivating learning activities.

\section{SOCIAL MEDIA TRAINING: PAST, PRESENT AND FUTURE TRENDS}

Social media training has quickly become mandatory for an ever-growing range of companies, far surpassing the wave of IT firms that embraced it in a first stage, like Dell, Intel and IBM (Meister and Willyerd, 2010). While it began as an added 'bonus' in the arsenal of the marketing spokesperson, now companies ranging from Unisys, PepsiCo, Adidas, HP and Sprint (http://www.sprint.com) are making social not only part of the company's core training curriculum, but also a key element in their recruiting message, stressing the employee benefit of receiving social media literacy training.

One obvious motivation for formalizing a company's social media program and policies is to avoid a social media disaster. The video entitled "Dirty Dominos Pizza" is an example of how dangerous the combination of social media and the workplace can be. The two Dominos Pizza employees who joked in the video while tampering with food, claimed afterwards that they were just having fun when they posted it to YouTube. As a result, both were fired and sent to jail on charges of food tampering. The consequences for the company of the incident are hard to erase: three years later, when a Google search on Domino's Pizza is made, the video is still the fifth result. A number of real episodes of this kind, damaging the image of companies, are continuously emerging and being noticed in the media in several countries and involving well known companies (Samsung, 2013; Fnac 2012).

Companies like Unisys, Sprint and HP are creating social media training programs to avoid these types of social media crises, but also, just as importantly, to show employees how using social media can be a valuable business tool which can increase their performance and productivity.

In fact, at a first stage, to avoid inappropriate online behaviour, most companies started by establishing specific social media guidelines, even though they would already have a policy of employee conduct. However, companies have found that guidelines were only the first step. They soon realized that they really needed a formal social media literacy program, which would offer a certification so that employees could share their progress and practices both on their employee directory as well as on LinkedIn, Facebook and even their Twitter profile. 
Taking the right steps in this arena can produce great benefits on the business side. As stated by Gloria Burke, Director of Knowledge \& Collaboration at Unisys, offering social media training creates a team of advocates who are equipped to represent their employer online. "When you give them that training, you're empowering them to be more confident and effective in what they're sharing...", Burke said. That means they will not only share news about their company but also feel confident they know how to do this in a safe and responsible manner while building their personal brand.

At Sprint, the social media-training brand is called Sprint Ninja, named after the type of warrior that originated in feudal Japan and specialized in unorthodox warfare. For its program, Sprint adopted many of the principles traditionally used to train Ninjas, like self control, moderation, and courtesy. Sprint employees who end up the Sprint Social Media Ninjas program (thus far, 2,400 volunteers have done so) complete a two-hour workshop to receive their Ninja certificate. Then, they become part of a community that is continuously engaged in discussion about how best to use social media to advocate for the company. Furthermore, Sprint is working to develop an online version of this training and offer it within Sprint University of Excellence.

Despite some initial arguments regarding the view that learning from experience is either the best or the only way to learn about social media, it is currently acknowledged that social media skills and abilities can be developed through training and that there is a need for formal education in social media. Given that the demand for social media skills and expertise is likely to rise much faster than available supply in the foreseeable future, we are likely to see an increase in courses and programs of all types (general training courses, specific training courses, certificate programs and degree offerings) as offerings in social media training and education (Hunt, 2014).

In this paper we put forward an extension to this view of social media training. Not only we take for granted that social media training is needed in organizations as we also propose that social media tools can be used with great benefits as a means to provide education and training to employees in the several business areas of the organization, ranging from human resources, to sales, marketing and customer care, to product development and production.
A well-known example of the feasibility of our view on this matter is the United Breaks Guitar case reported in (Ferreira et al., 2014) where a YouTube video posted by a very unsatisfied customer with United Airlines and which attracted millions of views was afterwards used by the company internally for training.

At this stage, we not only propose that social media can be used for training in organizations in an episodic way but also in a systematic way and as part of the organization training policy.

\section{THE EDUCATION AND TRAINING OF ORGANIZATIONAL WORKERS (ETOW) MODEL}

Social business in organizations is a reality that needs to be improved and systematized. Therefore, in Ferreira, Moreira \& Seruca (2014) the m_CSDT framework is proposed, which provides a way to introduce and/or systematize social business in organizations. In the following subsections, we describe a model - the EToW model - for the $2^{\text {nd }}$ layer of the $\mathrm{m}_{\text {CCSDT }}$ framework as well as a usage scenario for the model.

\subsection{The Rationale for the Model Proposal}

The relevance of conducting social business supported by social media tools is widely acknowledged and recognized by the scientific community and organizations, as discussed throughout the previous sections. However, there is a lack of approaches that allow the systematization and that guide its implementation within an organization. To address this end, an approach was proposed consisting of a three-layer framework (m_CSDT), covering the: (i) creation, sharing and the documentation of information and knowledge in and out of an organization; (ii) education and training of organizational workers, and (iii) promotion of ad-hoc discussion.

As shown in Figure 1, it is possible to develop a generic approach for implementing social business through the use of social media tools, in order to achieve the well-being of the organization considering the agility and collective intelligence dimensions. In the referred approach, emphasis is given in the production of value for the organizational ecosystem. 
The model shown in Figure 2 covers the second layer of the framework, called Education and Training of organizational Workers (EToW), which aims to use social tools to define educational and training strategies for the organization. It enables the conduction of training in the organization aligned with the organizational strategy, allowing training requested by the employees themselves or as a
The model identifies and presents the relationships and interactions between the organizational strategy, the training needs of the employees, social tools, training strategies and their implications for pratice and training in workpalce.

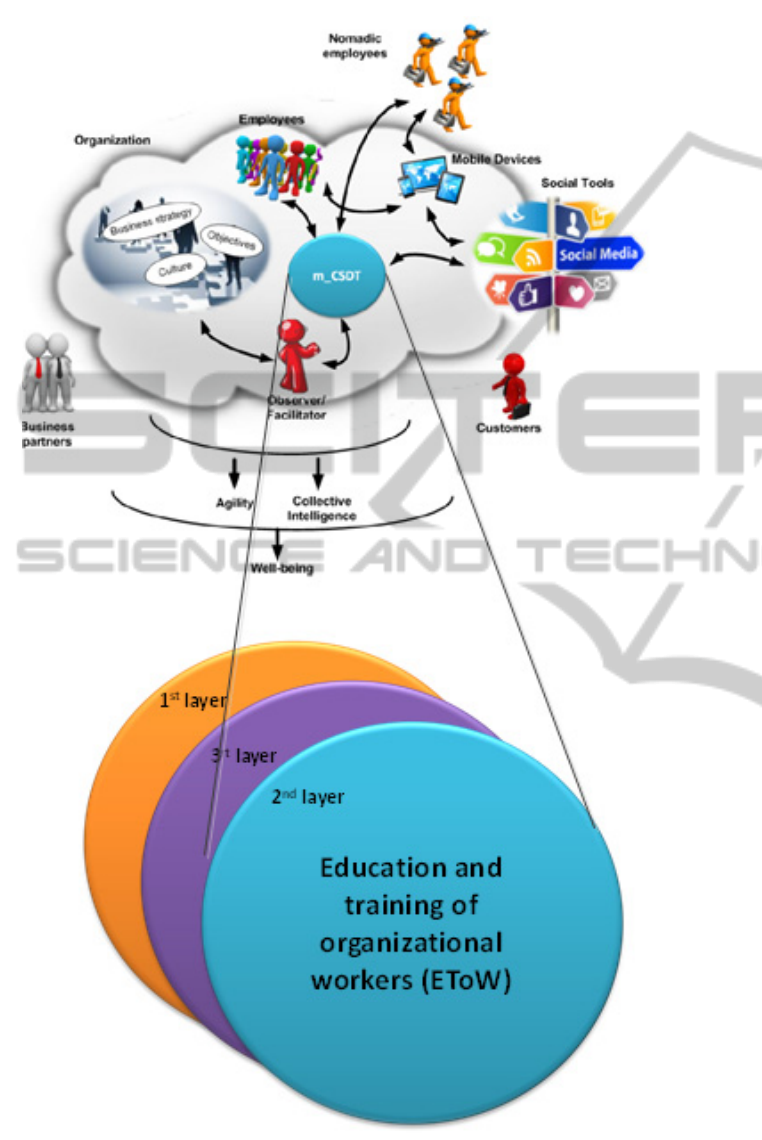

Figure 1: $2^{\text {nd }}$ layer - EToW - of the $\mathrm{m}_{-}$CSDT framework.

proposal made by the organization (of mandatory or optional attendance).

According to Figure 2, the EToW model of the $m$ CSDT framework aims the education and training of employees supported by social tools (4) (see section 3). Training strategies and training in the organization (2) are aligned with the organizational strategy (1). In order to allow a more flexible scheme of education and training, the model also allows the training proposed by the employees of the organization. Attending the training will be mandatory or optional, according to the strategy defined for training (3). The definition / adjustment of the training strategy (2) must be periodical so that it fits to the evolution / needs of the organization.

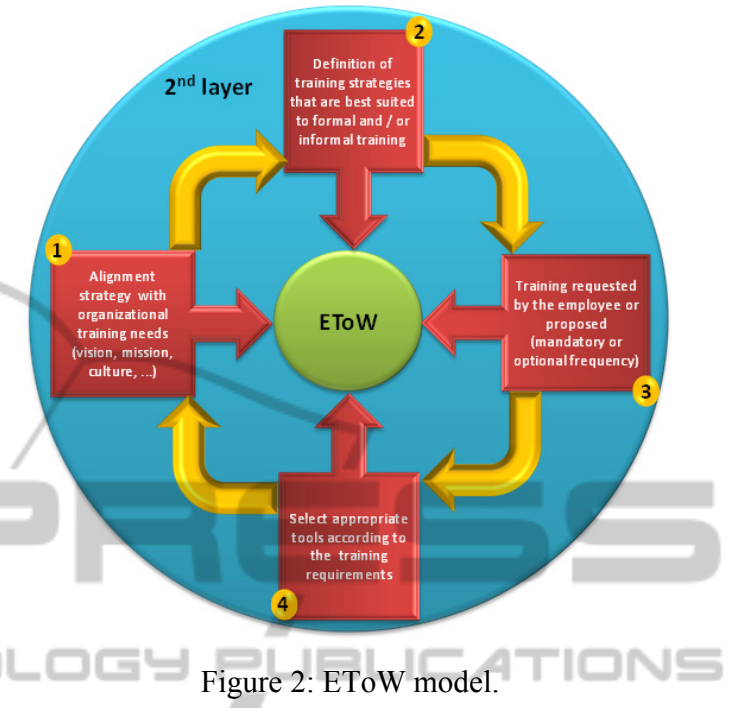

Regarding the use of Social Media tools, the model foresees a rather complete set of tools to be used within the training activities, which can be organized inside and outside the organization, i.e., in different learning contexts. We may assert that the model supports a learning classroom / distance, on the type of peer-to-peer mentoring or formal learning. In the learning type mentoring peer-topeer, the instructor acts as an observer / facilitator. whilest in the formal learning type, the instructor acts as an educator.

The use of social tools in learning is described in EToW as collaborative learning. That means that employees learn through participation and involvement with the (internal and external) social tools, connecting, interacting and collaborating to obtain or share information and / or knowledge through this kind of tools.

The use of social tools leads to the existence of sharing (implicit or explicit) of information on personal and professional networks. Thus, in order to overcome this potential problem (public exposure of information and knowledge owned by the organization), the creation of private groups is proposed, with restricted access to employees. Any violation of the established rules will be subject to sanctions.

The proposed model intends to promote the development of a culture of training and learning that supports lifelong training, adding value to the 
organizational development, as well as being central to achieve the objectives set in aligning organizational strategy with organizational training needs. Without a culture of learning incorporated into the ecosystem of the organization, it is unlikely that any training strategy in the organization may be successful.

\subsection{A Scenario for the Model Usage}

In this section, we outline a scenario for a possible training action that will incorporate social media tools - the $2^{\text {nd }}$ framework's layer. The scenario describes how Facebook, Twitter and YouTube, can be included in the training program.

At the beginning of the training program some accounts on Facebook and Twitter are created. Subsequently, a group is created on Facebook, where employees are invited to join. At this stage, it is expected that some inexperienced employees present some concerns related with the safety of the group, such as the addition of their profiles as well as the information that they are writing on Twitter. In response to these concerns it is possible to change the Facebook settings to allow access only to "friends" of the group's creator, who is the action's instructor. Regarding Twitter, only the instructor and other employees are allowed to follow. Furthermore, only one group member can send invitations to others to join the group. Thus, it is not possible for any other user of Facebook to access the group without an invitation. These training actions may involve employees who have never used this kind of tools, so a first action of training to learn how to use Facebook and Twitter effectively may be needed.

During the course of the training the instructor can put YouTube videos on the mural of the Facebook group created and learners are invited to comment the videos with their opinions. Simultaneously, trainees may ask any questions about the videos on the Facebook, as well as through Twitter by writing small messages. At this stage, there is no difference between employees who are in the organization and those who are on the move, as there is no difference in treatment and "equal" opportunities are given. If precise indications on how to make comments are not given, it is quite possible that in the early stages there are few answers and that the "Like" button is just used. One way to encourage the production of reviews is, for example, to assign a score grade to this activity so as to improve the final classification of the training action. During the training action, Facebook and Twitter can be used for the instructor to send messages to learners, tasks to be performed related to the action. What is expected is to have great interaction in the communication between the instructor and the trainees and between the trainees themselves.

It is expected that instructors will increase the number of questions posted on Facebook and Twitter on the next subjects taught, the types of assessments to be performed, deadlines for submission of tasks, among other tasks. This type of interaction will allow, for example, a collaborator who knows the answer to some question to "post" it even before the instructor realizes it, which can improve communication and a spirit of sharing and collaboration. It is also expected that as the action progresses more training videos are available in Youtube and their respective reviews increase in quantity and quality. It is believed that, at the end of the training action, the pedagogical use of Facebook, YouTube and Twitter has a positive impact on organizational learning through the use of mobile devices and with the contribution of the training group community, by creating, sharing and documenting information and knowledge.

At the end of the training action, a formal evaluation of the action will be carried out, through the use of a questionnaire and the results obtained will be compared with the results of training activities that do not use social media tools.

\section{CONCLUSIONS}

The $\mathrm{m}_{-}$CSDT framework referred in this paper aims to enable the implementation of social business in an organization. The approach is focused on the production of value for the customer, reducing whatever does not add value from his point of view as well as to the organization itself. Therefore, the involvement of the organization ecosystem is of paramount importance to achieve this goal. On the contrary, traditional approaches allow the conduction of business within an organization in a closed way.

In this paper we propose a model for the $2^{\text {nd }}$ layer of the framework in which social tools are used as a mean of transmission and exchange of knowledge, i.e., enabling the training of the organization's employees.

The model identifies the crucial role that an organization plays in a culture of their employees' learning and consequently contributes to achieving the organization's strategic objectives. 
Future work will include the testing of this model within a business environment, based on the experiences obtained with pilot tests performed under an academic context.

If any, should be placed before the references section without numbering.

\section{REFERENCES}

Anthony Myers (2013) McKensey Global Survey Finds Social Tools Tacking Hold in the Networked Enterprise, Retrieved August 02, 2013, from the http:// www.cmswire.com/cms/social-business/mackinse

Cara (2010) How Informal Learning Is Transforming the Workplace, Retrieved November, 2013, from http://www.caracorp.com/documents/CARA_SocialM ediaImpact PulseSurveyReport.pdf

Cortada, James, et al. (2012). The Business of Social Business: What works and how its done. IBM Institute for Business Value. Retrieved November, 2013, from the IBM website: http://www.ibm.com/midmarket/ common/att/pdf/IBV_2012_The_business_of_social_b usiness.pdf

Dunlap, J. C., \& Lowenthal, P. R. (2011). Learning, unlearning, and relearning: Using Web 2.0 technologies to support the development of lifelong learning skills. In G. D. Magoulas, E-infrastructures and technologies for lifelong learning: Next generation environments (pp. 292-315). London: University of London. Educause (2007). 7 things you should know about Twitter. Retrieved December, 2013, from http://www.educause.edu/ELI/7ThingsYouShouldKno wAboutTwitt/161801.

Ferreira, M. J., Moreira, F. \& Seruca, I. (2014). A traditional organization towards a new dimension of labour - social business. Handbook of Information systems and Technology for Organizational Agility, Intelligence, and Resilience, Hershey: Information Science Reference (IGI), (accept for publication).

Fnac (2012) FNAC revê campanha publicitária após queixas de clientes, http://expresso.sapo.pt/fnac-revecampanha-publicitaria-apos-queixas-de-clientes $=\mathrm{f} 70$ 2072

Friedrich, T., L., Vessey, W., B., Schuelke, M., J., Ruark, G., A. \& Mumford, M., D. (2009). A framework for understanding collective leadership: The selective utilization of leader and team expertise within networks. The Leadership Quarterly, 20, 933-958, DOI: 10.1016/j.leaqua.2009.09.008

Hagel, J., Brown, J. S., \& Davison, L. (2010). The Power of Pull: How Small Moves, Smartly Made, Can Set Big Things in Motion. New York: Basic Books.

Hunt C (2014) Social Media Education and Training: Where We Are, Where We're Going, http:// socialmediatoday.com/courtney-hunt/551223/ socialmedia-education-and-training-where-we-are-wherewe-re-going
IBM (2013). Social Business: Patterns in Achieving Social Business Success by leading and pioneering organizations. IBM Institute for Business Value. Retrieved November, 2013, from IBM website: http://social.bz/download/IBM-social-patterns.pdf

Jarche, H. (2011). Social learning for business. Retrieved November, 2013, from http://www.jarche.com /2011/01/social-learning-for-business/

Kim, P. (2012). The definition of social business. Retrieved from http://dachisgroup.com/2012/06/thedefinition-of-social-business/

Majchrzack, A., Cherbakov, L. \& Ives, B. (2009). Harnessing the Power of the Crowds with Corporate Social Networking "How IBM does it". MIS Quarterly Executive, 8(2):103-198.

Martin, A. \& van Bavel, R. (2103). Assessing the Benefits of Social Networks for Organizations. Retrived November, 2013 from http://ftp.jrc.es/EURdoc/ JRC78641.pdf

McKinsey\& Company (2012). The social economy: Unlocking value and productivity through social technologies, Retrieved November, 2013 from $\mathrm{http} / / /$ www.mckinsey.com/insights/high_tech_telecom s_internet/the_social_economy.

Meister J C, Willyerd K (2010) The 2020 Workplace: How Innovative Companies Attract, Develop, and Keep Tomorrow's Employees Today, Harper-Collins Publisher, New York.

Michelle Everson, Ellen Gundlach, Jacquelline Miller (2013) Social media and the introductory statistics course. Computers in Human Behavior. 29(5), A69A81. DOI: $10.1016 /$ j.chb.2012.12.033,

Mohammad Hossein Jarrahi \& Steve Sawyer (2013). Social Technologies, Informal Knowledge Practices, and the Enterprise, Journal of Organizational Computing and Electronic Commerce, 23:1-2, 110-

Samsung (2013) Campanha da Samsung gera indignação nas redes sociais, http://expresso.sapo.pt/campanha-dasamsung-gera-indignacao-nas-redes-sociais $=\mathrm{f} 778608$

Sideraworks (2013). What Is Social Business? Retrieved December, 2013, from http://www.sideraworks.com/ wp-content/uploads /2012/03/WhatIsSocialBusiness SideraWorks.pdf

Young, J. (2010). How social networking helps teaching (and worries some professors). The Chronicle of Higher Education, Retrieved July 18, 2013, from http://chronicle.com/article/How-Social-NetworkingHelps/123654/

Moore, R., Lopes, J., 1999. Paper templates. In TEMPLATE'06, 1st International Conference on Template Production. SCITEPRESS.

Smith, J., 1998. The book, The publishing company. London, $2^{\text {nd }}$ edition.

Yunus, M. (2007). Creating a World Without Poverty: Social Business and the Future of Capitalism. PublicAffairs, New York, USA

Wankel, L. \& Wankel, C. (2011). Connecting on campus with new media: Introduction to higher education administration with social media. in Cutting Edge Technologies in Higher Education, 2, xi-xviii. 